\title{
«Es gibt nichts Gutes, außer man tut es» - Arbeit an der Verständlichkeit von Gesetzestexten in der Schweizerischen Bundeskanzlei
}

\begin{abstract}
In recent years, some attempts have been made to establish guidelines for the drafting of statutory texts. None of these, however, have been very successful. Still, the need for such guidelines is felt. Over the last 25 years, the language sections of the Swiss Federal Chancellery (Sprachendienste der Schweizerischen Bundeskanzlei) have worked with optimising statutory texts. The article presents some of the principles, on which the work of the language sections is based, and underlines the importance of creating necessary institutional structures for optimising such texts. For optimisation can only be successful if language specialists are seriously involved in the whole drafting process.
\end{abstract}

\section{1. $\quad$ Einleitung}

Über die vermeintliche Schwerverständlichkeit von Gesetzestexten ${ }^{1}$ wird seit langem und immer wieder aufs Neue gesprochen und geschrieben, geklagt und geschimpft. Das Niveau dieser Diskussionen konnte in den letzten Jahren von den seichten Urgründen des Feuilletons doch um einiges angehoben werden. Die Schwärmerei vom allgemein verständlichen Gesetz und die pauschale Schuldzuweisung an die angeblich unwilligen oder unfähigen Juristinnen und Juristen macht allmählich einer

1 Natürlich greift die Problematik der Rechtsverständlichkeit weiter: Kommunikation und also Verstehensarbeit findet im Recht nicht nur über Gesetzestexte statt, sondern auch über die rechtlich strittigen Sachverhalte, sie findet statt zwischen Experten und Laien, zwischen Agenten und Klienten einer eigenartigen Institution usw.

* Markus Nussbaumer

Schweizerische Bundeskanzlei

Zentrale Sprachdienste

Gurtengasse 4

CH-3003 Bern

e-mail: markus.nussbaumer@bk.admin.ch 
fundierteren juristischen und linguistischen Theorie vom Gesetzestext, seinen Entstehungsbedingungen, seinen Funktionen und funktionsbedingten Formen, seiner Anwendung, seinen textsortenspezifischen Grenzen und den Möglichkeiten der Textoptimierung innerhalb dieser Grenzen Platz. ${ }^{2}$ Und auf der andern Seite wächst auch bei den Skeptikern, die bisher der Meinung waren, Spracharbeit an Gesetzestexten sei ohnehin nur Kosmetik und trage zur realen Rechtsarbeit überhaupt nichts bei, die Einsicht, mit richtig verstandener Gesetzestextoptimierung sei doch einiges zu gewinnen.

Noch immer reichlich schmalbrüstig ist das, was es an linguistischem Pflegeangebot für die Rechtsetzung gibt. ${ }^{3}$ Das Desiderat eines juristisch informierten linguistischen Leitfadens für die Gesetzesredaktion ist nach wie vor unerfüllt, aber die Chancen steigen, dass ein solcher heute entstehen könnte, auf dem festeren Boden der juristischen und linguistischen theoretischen Reflexion über Gesetzestexte und gespiesen aus einer langen und gründlichen Erfahrung der Praxis. Aber schon hier sei gesagt: Der allerbeste Leitfaden nützt wenig, wenn er nicht vermittelt wird in Aus- und Weiterbildung und vor allem wenn er nicht hineinwirken kann in eine Praxis, die eingebettet ist in geeignete institutionelle Rahmenbedingungen und in eine allgemeine Kultur verständlicher Gesetzessprache; nur in einem solchen Rahmen kann die Praxis die guten Ratschläge, von welcher Seite auch immer, aufnehmen und umsetzen.

In den Sprachdiensten der Schweizerischen Bundeskanzlei, in denen ich mitarbeite, wird seit nunmehr 25 Jahren intensiv Gesetzesredaktion betrieben, und erste Vorüberlegungen und Vorarbeiten für einen solchen Leitfaden für die Gesetzesredaktion sind in den letzten Jahren in Gang gekommen. Ich möchte im Folgenden ein paar Grundsätze unserer Verständlichkeitsarbeit und ein paar unabdingbare institutionelle Vorausset-

2 Erwähnt seien hier Hoffmann (1992), Busse (1992), Lötscher (1995), Sprache des Rechts [Themenheft] (2000) sowie die Nummer 20 (2001) des Rechtshistorischen Journals (Arena [Verständnis des Rechts] 2001); darin neben vielen anderen Nussbaumer (2001).

3 Vgl. Grewendorf (2000), der namentlich an den Fingerzeigen (1998) und am Handbuch der Rechtsförmlichkeit (1999) kein gutes Haar lässt - mit einigem Recht. Neuerdings hat sich auch die EU, gestützt auf eine Interinstitutionelle Vereinbarung vom 22. Dezember 1998 (Gemeinsame Leitlinien für die redaktionelle Qualität der gemeinschaftlichen Rechtsvorschriften; AB1. C 73 vom 17.3.1999, S. 1), einen Leitfaden für die Redaktion von Gesetzestexten (Gemeinsamer Leitfaden 2001) gegeben. 
zungen für eine erfolgreiche Gesetzestextoptimierung anführen. Ich illustriere den einen oder andern Punkt mit kurzen Beispielen aus unserer gesetzesredaktionellen Arbeit.

\section{Warum sind Gesetzestexte oftmals schwerer verständlich, als sie sein müssten?}

Das Wichtigste vorneweg: Gesetzestexte sind in aller Regel nicht deshalb unnötig schwer verständlich, weil die Gesetzesredakteure die Sprache nicht beherrschten. Das gibt es zwar auch, aber es ist das geringste Problem. Das größte Problem hingegen ist die Involviertheit der Gesetzesautoren in die Sache, ihr Verstricktsein in die Materie. Das führt zu Fachblindheit, zum Gefangensein in einer Behördenperspektive, die den Normadressaten nicht in seiner Perspektive abholt, es führt zu falschen Schwerpunktsetzungen, dass man also etwa vor lauter Details das Wichtigste zu sagen vergisst, weil es so selbstverständlich scheint. Es führt dazu, dass man vor lauter Bäumen den Wald nicht mehr sieht und nicht mehr abstrahieren kann und dass man aus Furcht vor Lücken überreguliert, in der falschen Hoffnung, die Zukunft ein für allemal normativ vorwegnehmen zu können, und damit den Gesetzestext zu einer dicken Haut werden lässt, die man über eine Wirklichkeit spannt, dass diese dahinter kaum mehr sichtbar wird. Ein Beispiel aus den Statuten der Eidgenössischen Versicherungskasse: In Artikel 20 soll die Höhe der Altersrente geregelt werden. Die Sachüberschrift - wichtiges verständnisleitendes Element - gibt das Thema vor, weckt die Frage, auf die der Normadressat nun die entsprechende Antwort sucht (in der linken Spalte der Entwurf aus dem Amt, in der rechten Spalte die Schlussfassung nach unserer redaktionellen Überarbeitung): 


\begin{tabular}{|c|c|}
\hline $\begin{array}{l}\text { Art. } 20 \text { Höhe der Altersrenten } \\
{ }^{1} \text { Die Altersrente richtet sich nach der Anzahl der } \\
\text { Versicherungsjahre und der vollendeten Altersjahre. } \\
\text { Sie beträgt } 60 \text { Prozent (Rentensatz) des versicherten } \\
\text { Verdienstes bei vollständiger Versicherungsdauer } \\
\text { von } 40 \text { Jahren und vollendetem } 62 \text {. Altersjahr. } \\
{ }^{2} \text { Wird die vorzeitige Ausrichtung der Altersrente } \\
\text { a. mit } 40 \text { und mehr Versicherungsjahren vor dem } \\
\text { vollendeten } 62 \text {. Altersjahr, } \\
\text { b. mit weniger als } 40 \text { Versicherungsjahren nach dem } \\
\quad \text { vollendeten } 62 \text {. Altersjahr, } \\
\text { c. mit weniger als } 40 \text { Versicherungsjahren vor dem } \\
\quad \text { vollendeten } 62 \text {. Altersjahr, } \\
\text { verlangt, so wird der Rentensatz versicherungsma- } \\
\text { thematisch gekürzt. Das EFD erlässt eine Tabelle } \\
\text { über die Kürzungssätze. } \\
{ }^{3} \text { Der Kürzungsfaktor für Fälle nach Absatz } 2 \text { Buch- } \\
\text { stabe c beinhaltet eine Kürzung wegen der fehlenden } \\
\text { Versicherungsjahre zusätzlich zur Kürzung für Vorbe- } \\
\text { zug vor dem 62. Altersjahr. }\end{array}$ & $\begin{array}{l}\text { Art. 20 Höhe der Altersrenten } \\
{ }^{1} \text { Die Altersrente beträgt höchstens } 60 \text { Prozent des } \\
\text { versicherten Verdienstes. } \\
{ }^{2} \text { Das Mitglied hat Anspruch auf die maximale Al- } \\
\text { tersrente, wenn es } 40 \text { Versicherungsjahre erreicht } \\
\text { und das 62. Altersjahr vollendet hat. Erfüllt es diese } \\
\text { Voraussetzungen nicht, so wird die Rente } \\
\text { entsprechend gekürzt. } \\
{ }^{3} \text { Das EFD erlässt eine Tabelle über die Kürzungssätze. }\end{array}$ \\
\hline
\end{tabular}

Im Ausgangstext wird in Absatz 1 das Wichtigste nicht explizit gesagt, es muss erschlossen werden: Die Altersrente beträgt höchstens 60 Prozent des versicherten Verdienstes. Mit dieser Norm fängt die Schlussfassung an.

Diese Maximalrente bekommt, wer zwei Bedingungen erfüllt; das ist die nächste Norm, und sie wird in der Schlussfassung in einem zweiten Absatz und als Anspruch des Berechtigten formuliert.

In Absatz 2 des Ausgangstextes hingegen folgt eine Kaskade von verwirrenden drei Fällen der Abweichung von den Voraussetzungen für die Höchstrente. Die Kaskade ist als Aufzählung in einen Rahmensatz eingebettet. Das widerspricht den gesetzestechnischen Richtlinien, die sich hier durchaus als sinnvoll erweisen, sind solche Aufzählungen mit anschließender Fortsetzung des Satzes doch in aller Regel sehr schwer zu verstehen. Die drei Typen sind aber ohnehin ein gänzlich überflüssiges theoretisches Konstrukt, das sich verunklärend vor eine ganz einfache Wirklichkeit schiebt: Wer die zwei Voraussetzungen für die Maximalrente nicht erfüllt, dem wird die Rente entsprechend der Nichterfüllung der Voraussetzungen gekürzt. Das kann man so einfach sagen, ohne das Geringste an normativem Gehalt zu verlieren. Verzichtet man auf die künstliche Typologie, so kann man auch ohne den geringsten Verlust auf Absatz 3 mit seinem Rückverweis auf Absatz 2 Buchstabe c verzichten. Hingegen sollte die Delegation der Kompetenz an das EFD (Eidgenös- 
sische Finanzdepartement), eine Tabelle mit den Kürzungssätzen zu erlassen, in einem eigenen dritten Absatz stehen.

Ein zweites Beispiel: In das Bundesgesetz über die politischen Rechte soll ein neues Kapitel eingeschoben werden; darin soll eine «Anrufinstanz» geschaffen werden, die die »Lauterkeit der politischen Werbung in Abstimmungskampagnen « zu beurteilen hat.

\begin{tabular}{|l|l|}
\hline Art. 82a Aufgabe & Art. 82a Grundsatz \\
& $\begin{array}{l}\text { Stimmberechtigte, die Aussagen im Rahmen politischer } \\
{ }^{1} \text { Die Anrufinstanz för die Eauterkeitder politischyn }\end{array}$
\end{tabular}

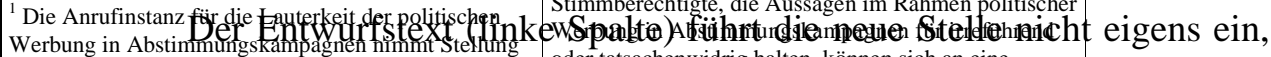

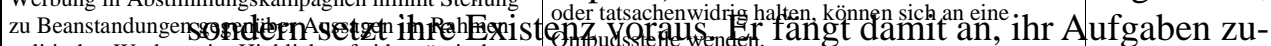
politischer Werbung im Hinblick auf eidgenössische

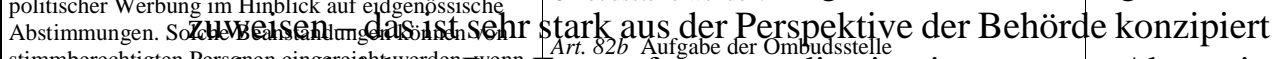
stimmberechtigten Persgnen eingereichtwerden, Wenn Art. $82 b$ Aufgabe der Ombudsstelle

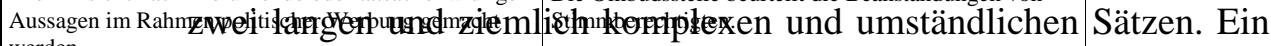

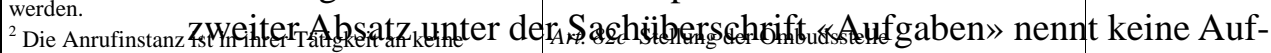
Weisungen gebundghabe, sondern formuliertrelemnfurdardsatzilendubabahängigkeit der Stelle.

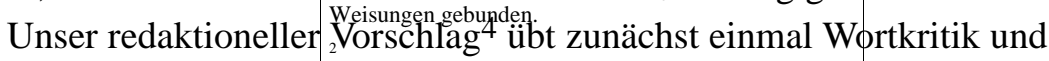
ersetzt den Ausdruck «Anrufinstanz» mit seinen religiösen Konnotationen durch den für solche Institutionen heute üblichen Ausdruck «Ombudsstelle». Was die Verständlichkeitsarbeit betrifft, ist das eher ein Neben-

4 Das Beispiel entstammt einer parlamentarischen Initiative aus dem Jahr 1999. Die Chancen stehen eher schlecht, dass dieser Gesetzestext je in Geltung erwachsen wird. Jedenfalls ist der Text heute noch nicht geltendes Recht, und es kann auch nichts darüber berichtet werden, ob unsere Vorschläge die Zustimmung der sachlich Zuständigen gefunden haben. Immerhin hat sich das Bundesamt für Justiz positiv über die Vorschläge geäussert. 
schauplatz. ${ }^{5}$ Gewichtiger ist die Umstellung im Ansatz: Da wir hier im Bundesgesetz über politische Rechte sind, sollte die neue Instanz als ein Recht der Stimmbürgerinnen und Stimmbürger eingeführt werden. Dies schlagen wir mit einem Artikel unter der Sachüberschrift «Grundsatz» vor. Wir gliedern stärker und setzen von diesem ersten Artikel einen zweiten Artikel ab, in dem wir der nun eingeführten Instanz die Aufgabe zuweisen. Daran schließen wir einen dritten Artikel an, in dem unter der Sachüberschrift »Stellung « unter anderem die Unabhängigkeit der Instanz von irgendwelchen Weisungen statuiert wird. Mit dieser Änderung in der Perspektive und der stärkeren Gliederung gelingt es auch, die Formulierungen kürzer und einfacher zu machen.

Eine Quelle für Schwerverständlichkeit ist darin zu sehen, dass Gesetzestexte manchmal in einem Zeitpunkt und aus einer Situation heraus formuliert werden müssen, da der politische Prozess noch nicht zu einer Lösung aller materiellen Fragen geführt werden konnte. Der Gesetzestext muss also Antworten vorgaukeln, wo wesentliche Fragen noch nicht beantwortet sind. Vor solchem Hintergrund kann kein befriedigender Gesetzestext entstehen. Die Redaktion kann diese Probleme natürlich nicht von sich aus lösen, sie kann aber solche «politischen Leichen» aufspüren und die Politik daran mahnen, dass sie ihre Arbeit gefälligst zu Ende zu führen hat.

Stärker in der Verfügungsmacht der Redaktion sind gesetzestechnische Unzulänglichkeiten, die oftmals die Verständlichkeit von Gesetzestexten stark behindern. Ein eher einfaches Beispiel haben wir oben bei den EVK-Statuten gesehen. Komplexer sind in der Regel etwa die Fälle falsch eingesetzter Legaldefinitionen - eine starke Tendenz gerade im EU-Recht. Ein besonders krasser Fall findet sich auch in der schweizerischen Medizinprodukteverordnung vom 24. Januar 1996, in deren Anhang 4 »Anforderungen an Präservative für Männer« aufgestellt werden. Ziemlich am Anfang dieses Anhangs findet sich folgende Definition von «Loch»:

Sichtbares Loch: Ein Loch im Präservativ, das bei normaler oder korrigierter Sehschärfe erkennbar ist.

Nichtsichtbares Loch: Ein Loch im Präservativ, das bei normaler oder korrigierter Sehschärfe nicht erkennbar ist und dadurch nachgewiesen wird, dass das Rollen eines mit 300 ml Wasser (oder Elektrolytlösung)

5 Selbstverständlich handelt es sich hier nicht um eine blosse Wortauswechslung; die damit einhergehenden Sinnverschiebungen wären zu diskutieren. 
gefülltes Präservativ auf gefärbtem Filtrierpapier sichtbare Spuren von

Nässe hinterlässt, wobei die gesamte Oberfläche des Präservativs mit dem Filtrierpapier in Berührung kommt.

Wir wissen doch, was ein Loch ist, auch was ein Loch in einem Präservativ ist, und auch die Vorstellung, dass Löcher so klein sein können, dass man sie von bloßem Auge nicht sieht, ist uns keineswegs fremd. Gerade darum befremdet es, wenn uns dieser selbstverständliche Begriff weggenommen und in verfremdeter Form zurückgegeben wird. Was soll das? Sucht man im weiteren Textverlauf, so findet man den Ausdruck «Loch« nur noch an einer einzigen Stelle: Unter der Sachüberschrift «Dichtheit» heisst es unter Ziffer 8 des Anhangs: »Bei der Prüfung muss für die Summe der sichtbaren und der unsichtbaren Löcher eine AQL von 0,25 erreicht werden. « Eine AQL ist eine »annehmbare Qualitätsgrenzlage«. Offenbar will man in diesem Anhang regeln, dass Präservative (mit einer gewissen Toleranz) dicht sein und dass sie entsprechend auf Löcher überprüft werden müssen und dass dafür kein einfacher Augenschein ausreicht, sondern ein bestimmtes Prüfverfahren anzuwenden ist. Statt dies an der geeigneten Stelle so zu sagen, wählt man den gesetzestechnischen Umweg über eine Legaldefinition - in diesem wie in vielen andern Fällen ein Holzweg für die Verständlichkeit.

Und schließlich kann man auch sprachlich das eine oder andere besser, sprich: einfacher, präziser, direkter machen. Auch dazu ein Beispiel (aus dem Umweltschutzgesetz):

\begin{tabular}{|c|l|}
\hline Art. 29c Vorschriften des Bundesrates & Art. 29c Vorschriften des Bundesrates \\
$\begin{array}{c}\text { Der Bundesrat kann überdies: } \\
\text { a. } \quad \begin{array}{l}\text { Vorschriften wie Anwendungsbeschrän- } \\
\text { kungen oder Verbote über den Umgang mit } \\
\text { bestimmten Organismen erlassen, wenn diese } \\
\text { den Menschen oder seine natürliche Umwelt } \\
\text { gefährden können; } \\
\text { Massnahmen wie Sanierungen gegen be- } \\
\text { die den Menschen oder seine natürliche Umwelt ge- } \\
\text { fährden können, einschränken, verbieten oder dafür } \\
\text { Sicherheitsmassnahmen vorschreiben. }\end{array} \\
\begin{array}{l}\text { stimmte Organismen vorschreiben, wenn } \\
\text { diese den Menschen oder seine natürliche } \\
\text { Umwelt gefährden können. }\end{array}\end{array}$ \\
\hline
\end{tabular}

Hier führt im Ausgangstext der Wille, zu gliedern, zu einer langen Wiederholung (»bestimmte Organismen, wenn diese ...«). Statt die betreffenden Organismen mit einem Relativsatz näher zu charakterisieren (»Organis- 
men, die ...«), werden sie mit einem Konditionalsatz umschrieben (»Organismen, wenn diese ...«). Eine Formulierung wie »Vorschriften wie Anwendungsbeschränkungen oder Verbote über den Umgang mit ... erlassen « ist umständlich und gerade in dieser Umständlichkeit unpräzis. Ein Gleiches gilt für die Formulierung »Massnahmen wie Sanierungen gegen ... vorschreiben«. Dasselbe lässt sich - siehe Fassung nach der Redaktion (rechte Spalte) - wesentlich kürzer, eleganter und präziser sagen.

\section{Unter welchen Voraussetzungen kann man die Verständlichkeit von Gesetzestexten verbessern?}

Die genannten Quellen für die Schwerverständlichkeit - die zu große Verstricktheit der Autoren in die Sache, politische Rücksichtnahmen, die mangelhafte Beherrschung der Textsorte (Gesetzestechnik), Formulierungsprobleme - sind wohl nicht grundsätzlich andere als bei jedem Schreiben. Aber natürlich fließen sie angesichts der Spezifik der Produktion von Gesetzestexten reicher, sprich: wirken diese generellen Verständlichkeitshindernisse stärker. Jedem Schreiben tut nach einer ersten Phase des Konzipierens und Formulierens der Blick von außen, durch einen unverstellten Leser gut. ${ }^{6}$ Genau dies muss auch in der Produktion von Gesetzestexten institutionalisiert werden: Die Verständlichkeit braucht einen Anwalt, der mit dem unvoreingenommenen Blick, soweit möglich von der Warte des künftigen Normadressaten her, liest, zu verstehen sucht, Fragen stellen und Vorschläge machen kann.

In der Schweiz ist in der Bundesgesetzesproduktion dieser Verständlichkeitsanwalt in der so genannten »Verwaltungsinternen Redaktionskommission « fest institutionalisiert. Folgende Merkmale zeichnen die Arbeit dieser Redaktionskommission aus:

- Die Kommission hat den unverstellten Blick von außen, da die Mitglieder der Kommission weder vom Fach sind noch die Vorphasen der politischen Auseinandersetzungen, der ersten Konzipierung und

6 Das wissen erfahrene Schreiberinnen und Schreiber, und sie wissen sich hier zu helfen. Das weiß die neuere Schreibdidaktik auf Grund der Schreibforschung und entwickelt deshalb auch neue Konzepte des Schreibunterrichts in der Schule, die allesamt wegkommen vom Schreiben als Prozess eines einzelnen in Einsamkeit und Freiheit und die das dialogische Moment in das Schreiben einzubauen suchen. 
der Formulierung mitgemacht haben und so als die ersten »Leser« an den Text herangehen können.

- Die Kommission ist interdisziplinär zusammengesetzt, besteht aus Linguisten (der Sprachdienste der Bundeskanzlei) einerseits und aus Juristen (der begleitenden Rechtsetzung des Bundesamtes für Justiz) andererseits; insbesondere mit den Linguisten ist in der Kommission ein gewisses Laienelement vertreten.

- Die Kommission muss zwingend konsultiert werden. Jeder Entwurf eines Gesetzes oder einer Rechtsverordnung muss über den Schreibtisch der Kommission und wird von ihr bearbeitet. Auch wenn die Arbeit der Redaktionskommission am Schluss in der Regel sehr geschätzt wird, ist sie doch oft zunächst einmal lästig, sie stellt Konzeption und Formulierungen in Frage, um die lange und entbehrungsreich gerungen wurde. Und wer lässt sich schon gerne an seinem Text herumflicken, und das von Leuten, die nicht vom Fach sind? Würde der Beizug der Redaktionskommission also auf Freiwilligkeit beruhen, so würde die Kommission vermutlich gerade bei denjenigen Entwürfen eher nicht konsultiert, die es besonders nötig haben, und die Einheitlichkeit der Gesetzeskultur litte enorm.

- Die Kommission muss im Verfahren früh konsultiert werden, wenn ein Projekt seine erste textuelle Kontur hat, der Text aber noch im Fluss ist, und sie begleitet einen Entwurf bis zur Verabschiedung.

- Die Kommission macht Vorschläge und stellt Fragen und ist damit ein wichtiger Spiegel für die Textautoren.

- Natürlich rührt gründliche Gesetzesredaktion auch an den materiellen Gehalt, und zwar nicht nur in dem Sinne, dass jede noch so kleine Umformulierung inhaltsrelevant werden kann, sondern so, dass man oftmals bewusst materielle Setzungen in Frage stellt, normative Konzepte umstößt und andere, einfachere, eingängigere vorschlägt. Anders gesagt: Wo der materielle Gehalt a priori Tabu ist, ist richtig verstandene Gesetzesredaktion nicht möglich.

- Die Fragen und Vorschläge werden anschließend mit den Autoren besprochen; im Dialog findet sich oftmals eine dritte Lösung, die dem Optimum näher kommt als der Entwurf und als der Gegenvorschlag der Redaktionskommission. In diesem Prozess des gemeinsamen Findens der Lösung können auch die für den Inhalt Verantwortlichen 
die materiellen Änderungen, die die Gesetzesredaktion angestoßen hat, akzeptieren.

- Falls sich die Kommission mit ganz wichtigen Anliegen beim zuständigen Amt nicht durchsetzen kann, hat sie die Möglichkeit, ihre Anliegen direkt in die Regierungssitzung zu tragen. Davon muss sehr selten Gebrauch gemacht werden, doch unterstreicht die Existenz dieses letzten Mittels den Stellenwert, den man in der Schweiz der Verständlichkeit beimisst.

- Als eine Besonderheit des dreisprachigen Schweizer Rechts muss noch erwähnt werden, dass Entwürfe von Gesetzen und wichtigen Verordnungen stets auf Deutsch und Französisch parallel ko-redigiert werden. Der Zwang, zugleich in zwei Sprachen eine möglichst klare, präzise und einfache Formulierung zu finden, wirkt sich positiv auf die sprachliche Qualität aus. Aber auch wenn ein Text nur in einer Sprache redigiert und dann in die andern übersetzt wird, ist dieser Übersetzungsprozess oftmals heilsam, wenn man bereit ist, das Original im Lichte der Übersetzung - dieser wohl intensivsten Form der Auseinandersetzung mit einem Text - noch einmal zu hinterfragen.

- Zusammenfassend: Die Verwaltungsinterne Redaktionskommission ist die institutionalisierte Multiplikation des Blicks von verschiedenen Seiten auf den entstehenden Text. In diesem mehrperspektivischen und dialogischen Verfahren läuft ein Klärungsprozess ab und klärt sich damit auch der Text.

\section{Ein Beispiel zum Schluss}

Ich schließe mit einem Beispiel, das noch einmal unsere Arbeit illustrieren soll. Links steht wiederum der Entwurf des Amtes; es handelt sich um einen neuen Artikel 27, der in das schon ältere Epidemiengesetz eingefügt werden soll. Rechts steht der Vorschlag unserer Redaktionskommission (der für einmal nicht ganz durchgedrungen ist - das gibt es natürlich auch): 
Bemerkungen

- Artikel 27 statuiert eine Meldepflicht; die Sachüberschrift kündigt das an. Auf diese Norm der Meldepflicht ist jedoch eine zweite Norm aufgepfropft, ein Rechtsetzungsauftrag an die Regierung, den Bundesrat, Ausführungsvorschriften zu dieser Meldepflicht zu erlassen. Diese zweite, sekundäre Norm deckt die erste und wohl »eigentliche « Norm

Art. 27 Meldepflicht der Meldepflicht 7
${ }^{1}$ Im Rahmen der Bekämpfung von übertragbaren

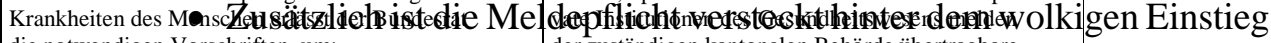

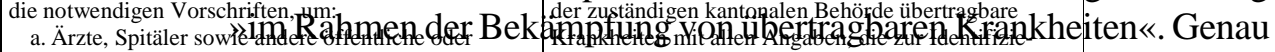

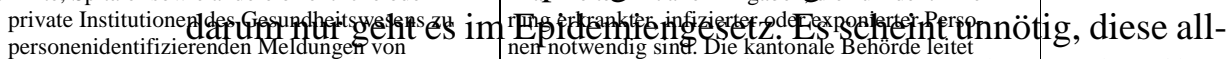

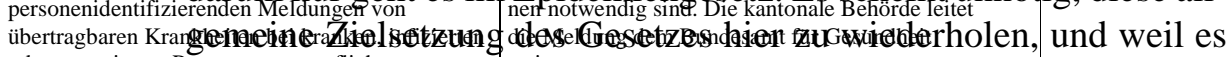

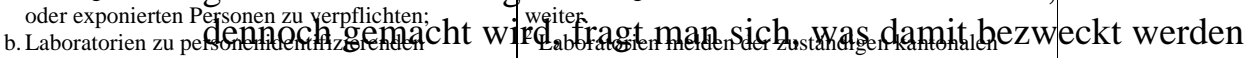

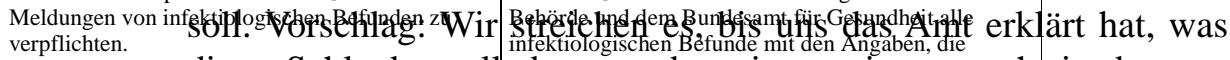

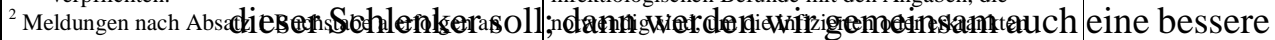
die zuständige kantonale Behörde, welche cie an das Personen zu identifizieren.

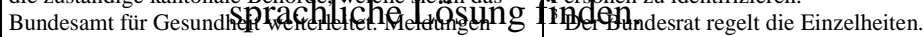

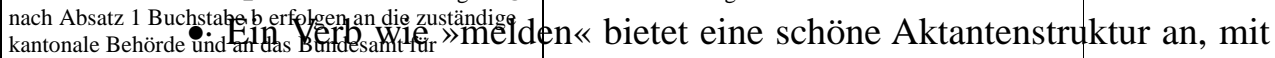
Gesundheit. der sich zwanglos eine verständliche Struktur bauen ließe: Wer muss wem was in welchem Umfang melden? Doch verhindert der Entwurf, dass sich diese Aktantenstruktur entfalten kann. Stattdessen erfindet er eine neues Wort: »personenidentifizierende Meldungen «, und der Adressat der Meldungen ist in einen separaten Absatz 2 mit Binnenrückverweisen ausgelagert.

- Mit dem Lösungsvorschlag formulieren wir die Meldepflicht wieder als Pflicht eines W, einem X ein Y im Umfang von Z zu melden. Wir 
machen dies in zwei Absätzen für zwei verschiedene Verpflichtete. In einem dritten Absatz folgt schließlich die untergeordnete Delegation an den Bundesrat, die Einzelheiten, wie dieser Meldepflicht nachzuleben ist, in einer Verordnung zu regeln.

Zum letzten Punkt: Man kann sich fragen, ob es diese Delegationsnorm wirklich braucht oder ob das nicht schon in der generellen Ausführungskompetenz der Regierung begründet ist. Natürlich ist das keine »rein redaktionelle Frage « im engeren Sinn, aber eine Frage, die sich gerade in der Redaktion immer wieder stellt und auch angegangen wird. Eine Redaktion, die sich solche Fragen entweder selbst verbietet oder verbieten lässt, verdient nicht, Gesetzesredaktion genannt zu werden.

\section{Internetadressen und Literatur}

http://www.admin.ch/ch/d/bk/sp/index.htm: Das ist die Homepage der Sektion Deutsch der zentralen Sprachdienste der Schweizerischen Bundeskanzlei. Hier finden sich unter anderem eine genauere Beschreibung der Verwaltungsinternen Redaktionskommission und ein Link auf DORES, die Dokumentation zu Recht und Sprache.

http://www.admin.ch: Das ist die Homepage der Bundesbehörden der Schweizerischen Eidgenossenschaft. Hier findet sich unter anderem ein Link zur Systematischen Sammlung des Bundesrechts, die sämtliche geltenden Gesetze und Verordnungen der Schweiz auf Bundesebene gratis abrufbar hält.

Arena [Verständnis des Rechts] (2001). In Rechtshistorisches Journal 20 (2001). 479729.

Busse, Dietrich (1992). Recht als Text. Linguistische Untersuchungen zur Arbeit mit Sprache in einer gesellschaftlichen Institution. Tübingen.

Fingerzeige (1998). Fingerzeige für die Gesetzes- und Amtssprache. Rechtssprache bürgernah. 11. Auflage, neu bearb. u. aktualisiert von Ulrich Daum. Hrsg. v. der Gesellschaft für deutsche Sprache, im Einvernehmen mit dem Bundesministerium des Innern und dem Bundesministerium der Justiz. Wiesbaden.

Gemeinsamer Leitfaden (2001). Gemeinsamer Leitfaden für Personen, die in den Gemeinschaftsorganen an der Abfassung von Rechtstexten mitwirken. Brüssel.

Grewendorf, Günther (2000). Die sprachliche Pflege des Rechts. Linguistische Aspekte der Rechtsprüfung. In Sprache des Recht [Themenheft]. Hrsg. v. Rainer Dietrich und Wolfgang Klein. Zeitschrift für Literaturwissenschaft und Linguistik (LiLi) 118/2000. 96-114. 
Handbuch der Rechtsförmlichkeit (1999). Empfehlungen des Bundesministeriums der Justiz zur einheitlichen und rechtsförmlichen Gestaltung von Gesetzen und Rechtsverordnungen nach $\S 38$ Abs. 3 GGO II. 2., neu bearb. Aufl. Köln.

Hoffmann, Ludger (1992). Wie verständlich können Gesetze sein? In G. Grewendorf (Hg.): Rechtskultur als Sprachkultur. Frankfurt/M. 1992. 122-154.

Lötscher, Andreas (1995). Der Stellenwert der Verständlichkeit in einer Hierarchie der kommunikativen Werte für Gesetze. In Richard J. Watts/Iwar Werlen (Hrsg.): Perspektiven der angewandten Linguistik. Neuchâtel (= Bulletin suisse de linguistique appliqué 62), 109-127.

Nussbaumer, Markus (2001). Von Schwärmern und Skeptikern und ein Versuch, Realist zu sein - Bilanz und Entwurf des Sprachspiels vom unverständlichen Gesetz. In Rechtshistorisches Journal 20 (2001). 644-655.

Sprache des Rechts [Themenheft] (2000). Hrsg. v. Rainer Dietrich und Wolfgang Klein. Zeitschrift für Literaturwissenschaft und Linguistik (LiLi) 118/2000. 
